\title{
Mesenchymal stem cell-mediated suppression of hypertrophic scarring is p53 dependent in a rabbit ear model
}

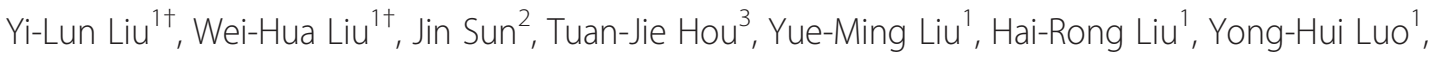 \\ Ning-Ning Zhao', Ying Tang ${ }^{2}$ and Feng-Mei Deng ${ }^{2^{*}}$
}

\begin{abstract}
Introduction: Mesenchymal stem cells (MSCs) are considered to play important roles in wound repair and tissue remodeling. Hypertrophic scar (HTS) is a cutaneous condition characterized by deposits of excessive amount of collagen after an acute skin injury. However, currently there is little knowledge about the direct relationship between MSCS and HTS.

Methods: The hypertrophic scar model was established on rabbit ears. MSCs were isolated from rabbit femur bone marrow and transplanted through ear artery injection. Hypertrophic scar formation was examined using frozen-section analysis, hematoxylin and eosin (HE) staining, Masson's trichrome staining, and scar elevation index. The role of p53 in the MSCs-mediated anti-scarring effect was examined by gene knockdown using p53 shRNA.

Results: In this study, MSCs engraftment through ear artery injection significantly inhibited the hypertrophic scarring in a rabbit ear hypertrophic scar model, while this anti-scarring function could be abrogated by p53 gene knockdown in MSCs. Additionally, we found that MSCs down-regulated the expression of TGF- $\beta$ receptor I (TRRI) and alpha-smooth muscle actin (a-SMA) at both mRNA and protein levels in a paracrine manner, and this down-regulation was rescued by p53 gene knockdown. Moreover, our results showed that MSCs with p53 gene knockdown promoted the proliferation of fibroblasts through increasing nitric oxide (NO) production.
\end{abstract}

Conclusions: These results suggest that MSCs inhibit the formation of HTS in a p53 dependent manner through at least two mechanisms: inhibition of the transformation of HTS fibroblast to myofibroblast; and inhibition of the proliferation of fibroblasts through inhibition of NO production.

\section{Introduction}

Hypertrophic scar (HTS) is a common complication of burn injury and other soft tissue injuries. Cosmetic and functional impairment caused by HTS remains a great challenge to burn and plastic surgeons [1,2]. HTS is characterized by the proliferation of a large number of fibroblasts, accumulation of collagen and infiltration of inflammatory cells [3]. Apart from the fibroblasts that have been recognized as one of the driving factors of HTS, mesenchymal stem cells (MSCs) were found to have multiple functions in the formation of HTS [3-5].

\footnotetext{
* Correspondence: thirdforce@163.com

${ }^{\dagger}$ Equal contributors

2Department of Pathology, Chengdu Medical College, 783 Xindu Avenue, Xindu District, Chengdu 610500, China

Full list of author information is available at the end of the article
}

MSCs are multipotent cells that can migrate to the wound sites, where they form part of the microenvironment [6-8], improve wound healing and inhibit hypertrophic scarring [9-11]. In addition to the differentiation potential, MSCs can interact with many kinds of cells in the microenvironment through paracrine signaling pathways [12,13]. Activated MSCs can produce abundant oxidizing agents such as nitric oxide (NO) and cytokines, through which MSCs potently suppress immune responses and influence tumor cell proliferation and phenotype transformation in the tumor microenvironment $[14,15]$. However, the mechanisms of the anti-scarring function of MSCs and the interaction between MSCs and HTS fibroblast remain poorly understood.

Pathological scar is considered a tumor-like tissue structure exhibiting an uncontrolled growth manner following 
wound healing. As one of the most intensively studied tumor-suppressor genes, p53 is also involved in the formation of pathological scar including HTS [16,17]. An elevated p53 protein level was detected in HTS tissue, compared with normal scar or atrophic white scar [18], but the exact functions of p53 in the scar formation are still not clear.

Recently, studying the roles and mechanisms of stromal cells in tumor formation is a popular field. One study showed that the p53 gene status in tumor-infiltrating MSCs influenced the development of tumor [12]; thus it is an interesting question whether the p53 gene status in MSCs can influence HTS formation. A better understanding of the roles of p53 gene status in the stromal cells would probably provide important insight into HTS pathogenesis. In the present study, we examined the contribution of p53 in MSCs to HTS formation by administering MSCs with or without p53 stable knockdown into rabbit ear HTS models. HTS formation was examined by frozen-section analysis, hematoxylin and eosin (HE) staining and Masson's trichrome staining, and was evaluated using the scar elevation index (SEI). Our results showed that wild-type MSCs exerted an anti-scarring effect on the HTS model, but p53-deficient MSCs had little influence on the development of HTS. Instead, p53-deficient MSCs resulted in scar recurrence compared with wild-type MSCs. Further study showed that p53 knockdown abrogated the capability of MSCs to inhibit the transformation of HTS fibroblast to myofibroblast. Moreover, p53 deficiency in MSCs resulted in higher NO production, which may promote HTS fibroblast proliferation. Taken together, our study revealed an important role for p53 in MSCs during wound healing and the HTS formation process.

\section{Methods \\ Reagents}

Puromycin, $\quad N^{\mathrm{G}}$-monomethyl-L-arginine acetate salt (L-NMMA) and Griess reagent were from Sigma-Aldrich (St. Louis, MO, USA). Rabbit p53 shRNA lentiviral particles, control shRNA lentiviral particles and rabbit polyclonal antibody against p53 were purchased from Abiocode Bio-Technology (Shanghai, China). Fluorescein isothiocyanate-conjugated antibodies against CD45 and CD90 and phycoerythrin-conjugated antibodies against CD34 and CD105 were from BD Biosciences Pharmingen (San Diego, CA, USA). Dexamethasone, ascorbic acid and $\beta$-glycerophosphate were from SigmaAldrich Chemicals (St. Louis, MO, USA). MesenCult medium with adipogenic stimulatory supplements was from StemCell Technologies (Vancouver, BC, Canada). The mouse monoclonal antibody against CD31 and the rabbit polyclonal antibodies against transforming growth factor-beta receptor 1 (T $\beta R \mathrm{R}$ ) and alpha-smooth muscle actin ( $\alpha$-SMA) were from Santa Cruz Biotechnology (Santa Cruz, CA, USA). Recombinant rabbit interferon gamma and tumor necrosis factor alpha (TNF $\alpha)$ were from eBiosciences (La Jolla, CA, USA).

\section{Ethics statement, rabbits and cell culture}

This study was approved by Animal Experimentation Ethics Committee of the First Afiliated Hospital of Chengdu Medical College, China. All animal treatments were carried out in accordance with Guidelines on Care and Use of Laboratory Animals issued by the Chinese Council on Animal Research and the Guidelines of Animal Care.

New Zealand white rabbits were purchased from Experimental Animal Center of Sichuan Province, with an initial body weight of $2.0 \pm 0.3 \mathrm{~kg}$ (for the rabbit ear model of hypertrophic scarring). Animals were housed in a regulated environment $\left(22 \pm 2^{\circ} \mathrm{C}\right)$, with a 12-hour light/dark cycle (light cycle was from 08:00 to 20:00). All animals were fed according to the Specific Pathogen Free Animal Criteria.

MSCs were generated from femur bone marrow using New Zealand white rabbits ( 1 month old, male) by the holo-bone marrow adherence method. In brief, the bone marrow fluids were extracted under aseptic conditions and transferred into tube with isopyknic Hanks liquid, and centrifuged at $1,000 \mathrm{rpm}$ for 5 minutes at $4^{\circ} \mathrm{C}$. After removing supernatant, cells were washed with Hanks liquid and cultured in Dulbecco's modified Eagle's medium supplemented with $10 \%$ fetal bovine serum, $2 \mathrm{mM}$ glutamine, $100 \mathrm{U} / \mathrm{ml}$ penicillin and $100 \mu \mathrm{g} / \mathrm{ml}$ streptomycin (Invitrogen, Carlsbad, CA, USA). Nonadherent cells were removed 8 hours later, and adherent cells were maintained with medium replenishment every 3 days. The cells reached $90 \%$ confluence after 14 to 16 days, and were passaged at a split ratio of $1: 3$ to 5 . The cells of passages 4 to 6 were used for experiments. Lineage cell surface markers (CD34, CD45, CD90, and CD105) were detected using flow cytometry (BD Accuri C6; Becton Dickinson San Jose, CA, USA).

HTS fibroblasts were generated from the rabbit ear HTS tissue. In brief, the rabbit ear HTS tissue was cut into $0.5 \mathrm{~mm}$ pieces and digested with $0.25 \mathrm{~g} / \mathrm{l}$ dispase II. The dermal tissue was minced and digested with $30 \times$ volume of collagenase I $(200 \mathrm{U} / \mathrm{ml})$ at $37^{\circ} \mathrm{C}$ for 2 hours, followed by centrifugation. The cells were collected and cultured in Dulbecco's modified Eagle's medium containing $15 \%$ fetal bovine serum at $37^{\circ} \mathrm{C}$ in $5 \%$ carbon dioxide. The culture medium was changed every 3 days. At 80 to $90 \%$ confluence, cells were passaged and cultured in Dulbecco's modified Eagle's medium containing 10\% fetal bovine serum. The cells of passages 4 to 6 were used for further experiments. 
The method of isolating engrafted MSCs from scar tissue is the same as for HTS fibroblast isolation [19]. Ten days after isolation, the cells in six-well plates were observed using fluorescent microscope. The cells that show a green fluorescence signal are engrafted MSCs, while the cells without a green fluorescence signal are HTS fibroblasts. The number of fluorescent cells/total cells was calculated in five randomly selected microscopic fields $(\times 100)$ of each well. Three scar tissues from each group were examined.

\section{Flow cytometry analysis}

Cells were trypsinized, washed with phosphate-buffered saline, and incubated with antibodies against CD34, CD45, CD90 and CD105. Flow cytometry detection was performed with a BD Accuri C6 flow cytometer (Becton Dickinson).

\section{Osteogenic induction and adipogenic induction}

For osteogenic induction, cells with $70 \%$ confluence were incubated in osteogenic medium supplemented with $10^{-7} \mathrm{M}$ dexamethasone, $0.2 \mathrm{mM}$ ascorbic acid and $10 \mathrm{mM} \beta$-glycerophosphate. The medium was changed twice a week. Cell colonies displayed bone-like nodular aggregates of matrix mineralization after 14 days in differentiation medium. The cells were stained with alizarin red. For adipogenic induction, MesenCult medium with adipogenic stimulatory supplements was used to treat the cells. The medium was changed every 3 to 4 days. The cells were stained with oil red after culturing for 21 days.

\section{Lentivirus transduction}

Rabbit bone marrow MSCs were transduced by lentiviral particles containing rabbit p53 shRNA or control shRNA for 24 hours, and then subjected to puromycin $(3 \mu \mathrm{g} / \mathrm{ml})$ selection. Stable clones were harvested 2 weeks later, and passaged with normal media.

\section{Western blot}

Cells were lysed in lysis buffer containing phenylmethylsulfonyl fluoride, leupeptin and other protease inhibitors for 1 hour on ice. Protein samples were separated on sodium dodecyl sulfate-polyacrylamide gels, then transferred onto polyvinylidene difluoride membranes, and incubated with corresponding primary antibodies at $4^{\circ} \mathrm{C}$ overnight. After washing, membrane was incubated with secondary antibodies, and subjected to chemiluminescent detection according to the manufacturer's instructions.

\section{3-(4,5-dimethylthiazol-2-yl)-5(3-carboxymethonyphenol)-} 2-(4-sulfophenyl)-2H-tetrazolium (MTS) assays

Cells were seeded into flat-bottomed 96-well culture plates at 5,000 cells/well, and grew for 48 hours. Cells were labeled with the VisionBlue reagent and detected by Synergy 2 multidetection microplate reader (BioTek, Winooski, VT, USA) according to the manufacturer's instruction.

\section{Colony formation assay}

Cells were seeded at 2,000 cells/well onto six-well culture plates and allowed to grow for 12 days before fixation with methanol and staining with crystal violet ( $0.5 \%$ solution $)$.

\section{Rabbit ear model of hypertrophic scarring, grouping and MSC engraftment}

The rabbit ear model of hypertrophic scarring was established according to the procedure previously described by Kryger and colleagues with a minor modification [20]. In brief, rabbits were intravenously anesthetized with sodium pentobarbital $30 \mathrm{mg} / \mathrm{kg}$. Under aseptic operation conditions, four round wounds each with a diameter of $9 \mathrm{~mm}$ were created down to the bare cartilage on the ventral surface of each ear along the long axis. The epidermis, dermis and perichondrium in each wound were thoroughly removed, and the wounds were covered with sterile gauze for 1 day. After this operation, the rabbits were provided with conventional anti-inflammatory treatment. Three weeks after operation, the healed wound surface showed an obvious protrusion, an indicator of HTS formation. Five weeks post operation, the formation of HTS reached the peak level.

Twelve rabbits were divided into three groups with age and gender matched: control group; control shRNA MSC group; and p53 shRNA MSC group. Three days after the model operation, $1 \times 10^{5}$ cells of control shRNA MSCs or p53 shRNA MSCs (in $1 \mathrm{ml}$ phosphate-buffered saline) were injected into the rabbits via ear artery once every 2 days up to eight times. Phosphate-buffered saline was used in the control group.

\section{General observation and histological analysis}

General observations including growth status, color, texture and thickness of HTS were recorded by photographic imaging at designated time points. In addition, the height of HTS was measured.

Full-thickness biopsies of the wound-repair bed and surrounding tissue were obtained at 3, 4 and 5 weeks after operation. The tissues were subjected to frozensection analysis. Paraffin sections were subsequently subjected to HE staining, and Masson's trichrome staining using Masson's Trichrome Stain Kit (KeyGen Biotech Co., Nanjing, China) following the manufacturer's instructions. In brief, the sections were stained in Weigert's iron hematoxylin working solution for 10 minutes and in Biebrich scarlet-acid fuchsin solution for 5 minutes, and then placed in 1\% phosphomolybdic-phosphotungstic acid solution for 5 minutes. The sections were then stained in aniline blue solution for 5 minutes, and rinsed 
in $1 \%$ acetic acid solution for 2 minutes. Collagen fibers were stained blue. Keratin and muscle fibers were stained red. Cell cytoplasm and nuclei were stained light pink and dark brown, respectively. In each section of Masson's trichrome staining, three high-power fields in the dermis of wound area or unwounded normal tissue, inferiorly by the ear cartilage and superiorly by the epithelial basement membrane, were randomly selected and photographed. The integrated optical density analysis of Masson's trichrome staining was performed by Image-Pro Plus 6.0 software (Media Cybernetics, Silver Springs, MD, USA) [21].

The SEI, which measures the ratio of total scar connective tissue area to the area of underlying dermis, was evaluated using Image-Pro Plus 6.0 software. The height of the underlying dermis was determined based on the height of the adjacent unwounded dermis. An SEI of 1 indicated no newly formed hypertrophied dermis, whereas SEI >1 denoted HTS formation [22]. CD31 in the scar tissues was detected by immunohistochemistry using monoclonal anti-CD31 antibody (1:100).

\section{Real-time PCR}

Total RNA was isolated using RNAprep pure Cell/ Bacteria Kit (Tiangen Biotech, Beijing, China), and firststrand cDNA synthesis was performed using the 1st cDNA Synthesis Kit with oligo $(\mathrm{dT})_{15}$ (Tiangen Biotech). The mRNA levels were measured by real-time PCR using SYBR Green Master Mix (Roche Diagnostics, Indianapolis, IN, USA). The total amount of mRNA was normalized to endogenous $\beta$-actin mRNA.

Sequences of PCR primer pairs were as follows: T $\beta R I$, forward primer 5' ${ }^{\prime}$ TGTTGGTACCCAAGGAAAGC-3' and reverse primer $5^{\prime}$-CACTCTGTGGTTTGGAGC AA-3'; $\alpha$-SMA, forward primer 5'-CAGGGAGTAATG GTTGGAAT-3' and reverse primer $5^{\prime}$-TCTCAAACATAATCTGGGTCA-3'; inducible nitric oxide synthase (iNOS), forward primer 5'-CAGCTGGGCTCAACAA ACCTT-3' and reverse primer 5'-CATTGGAATGGA AGCGTATCG-3'; and $\beta$-actin, forward primer $5^{\prime}-\mathrm{GC}$ TATTTGGCGCTGGACTT-3', and reverse primer $5^{\prime}$ GCGGCTCGTAGCTCTTCTC-3'.

\section{Detection of nitric oxide}

NO was detected using a modified Griess method. Briefly, all $\mathrm{NO}_{3}^{-}$was converted into $\mathrm{NO}_{2}^{-}$by nitrate reductase, and total $\mathrm{NO}_{2}^{-}$was detected by the Griess reaction [23].

\section{Statistical analysis}

SPSS Version 13.0 for Windows (SPSS Inc., Chicago, IL, USA) was used for data analysis. Data were presented as the mean \pm standard error of the mean. Statistical significance was assessed by one-way analysis of variance. Significance was set at $P<0.05$.

\section{Results}

\section{Establishment of the p53 stable knockdown cell line of} rabbit MSCs

Passage 4 of MSCs was transduced with lentiviral particles containing p53 shRNA or control shRNA, each with a green fluorescent protein label. After transduction, cells were selected with puromycin, then passaged for further use (Figure 1A). p53 knockdown was successfully achieved as shown by western blot. The expression of p53 protein decreased more than $80 \%$ (Figure 1B,C). 3-(4,5-dimethylthiazol-2-yl)-5(3-carboxymethonyphenol)-2-(4sulfophenyl)-2H-tetrazolium (MTS) and colony formation assays were performed to test the cell viability and colony formation capacity of p53 shRNAtransduced or control shRNA-transduced cells. There was no significant difference between the two groups on 3-(4,5-dimethylthiazol-2-yl)-5(3-carboxymethonyphenol)-2-(4-sulfophenyl)-2H-tetrazolium (MTS) and colony formation (Figure 1D,E,F). CD90 and CD105 were highly expressed in both p53 shRNA-transduced and control shRNA-transduced MSCs. CD34 and CD45 expressions were very low in the above cells (Figure 1G). Both kinds of cells would differentiate into mineralizing cells stained by alizarin red after osteogenic induction or adipocytes stained by oil red after adipogenic induction (Figure $1 \mathrm{H}$ ). These results suggested that p53 shRNA-transduced and control shRNA-transduced MSCs maintained the common features of MSCs.

\section{Exogenous MSCs migrated into rabbit ear hypertrophic scar tissue by transplantation via ear artery}

Endogenous MSCs are known to be recruited to the sites of damaged tissue, while exogenous MSCs have also been shown to migrate to target tissue by transplantation via associated artery or by bone marrow transplantation [12,24]. In this study, to examine whether exogenous MSCs were recruited into the HTS tissue after transplantation via ear artery, MSCs with or without p53 stable knockdown, both with a green fluorescent protein label, were injected into the rabbit ear via the ear artery during the wound healing process in the rabbit ear HTS model. Scars were excised and subjected to frozen-section analysis and HE staining 4 weeks after model operation. The green fluorescence in the frozen sections indicated the presence of the transplanted MSCs that migrated into the scar tissue (Figure 2A). MSCs were also isolated from the scar tissue and cultured in the media. A fraction of the scar tissueinfiltrated MSCs expressed green fluorescent protein in both p53 shRNA-transduced and control shRNAtransduced cells $(14.8 \% \pm 0.9$, and $15.3 \pm 0.7$ respectively) (Figure 2B). 


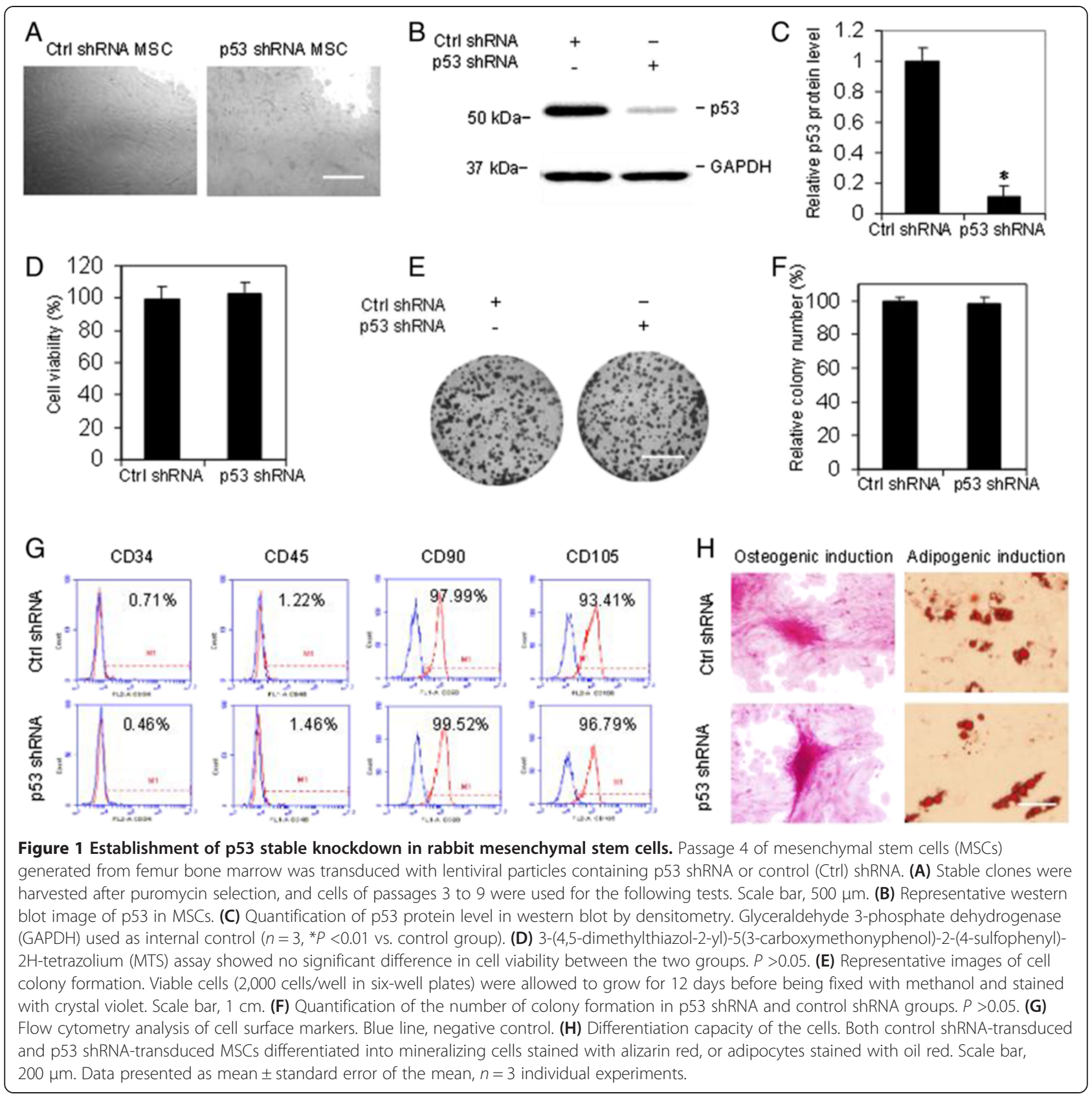

p53 is essential for MSCs to prevent the progression of hypertrophic scar formation in the rabbit ear model

The control group without MSC treatment showed successful HTS formation after operation characterized by obvious hyperplasia, collagen fiber accumulation and abundant blood vessels, according to the general observations and histological analysis (Figure 3A,B,C,D, first panel). MSCs transduced with control shRNA inhibited the HTS formation (Figure 3A,B). The SEI was significantly reduced in control shRNA-transduced MSCs group at 3, 4 and 5 weeks after model operation compared with control group without MSCs and the p53 shRNA-transduced MSC group (Figure 3E). The control shRNA-transduced MSC group showed less collagen deposition, indicated by lighter Masson's trichrome staining (Figure 3C,F) and less CD31-positive blood vessels (Figure 3D) by immunohistochemistry compared with the control group without MSCs and the p53 shRNA-transduced MSC group. However, there was no significant difference in the SEI and histological results between the p53 shRNA-transduced MSC group and the normal control group. Taken together, the results indicated that MSCs significantly inhibited the development of HTS and that p53 knockdown abrogated the effects of 

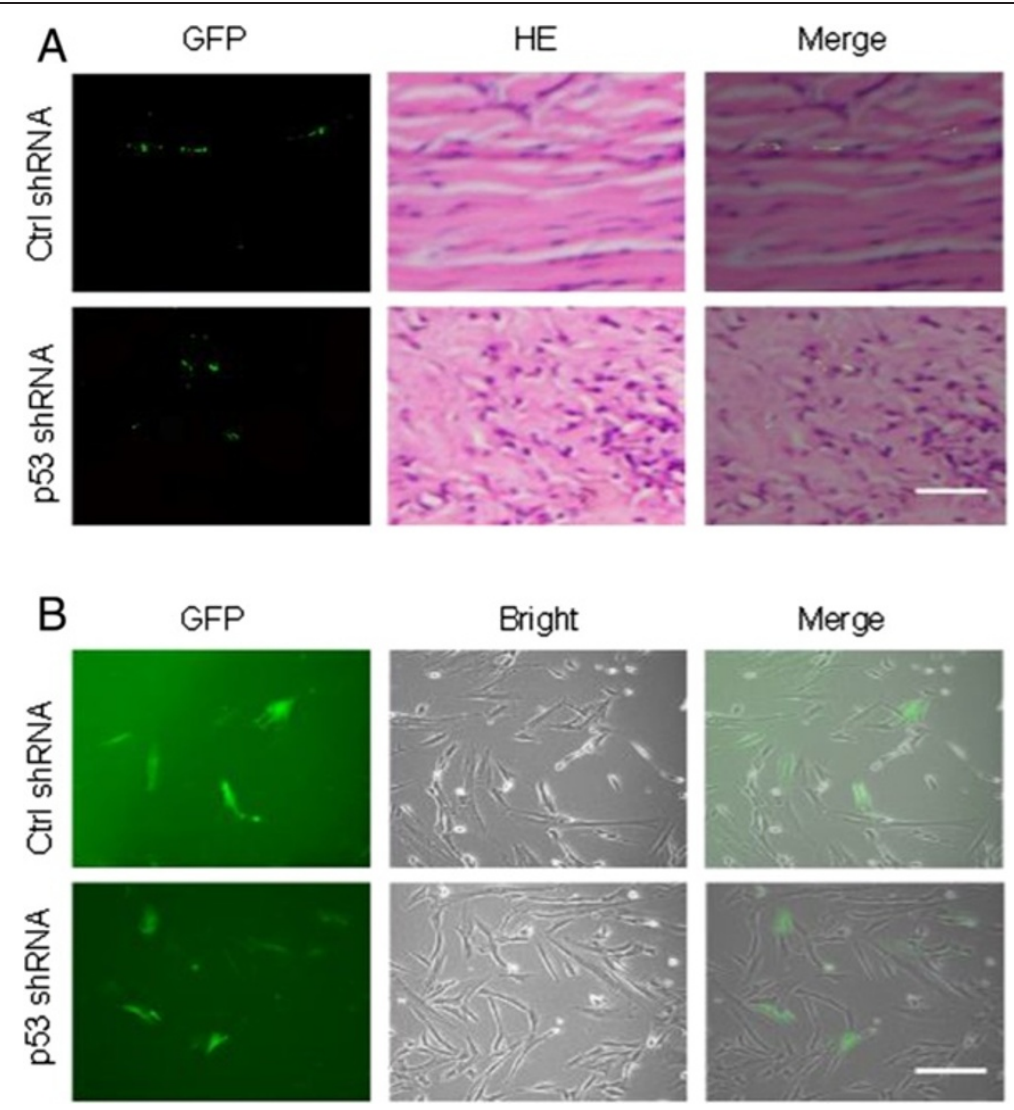

Figure 2 Mesenchymal stem cells with or without p53 stable knockdown, both with green fluorescent protein label, were injected via ear artery during the wound healing process of the rabbit ear hypertrophic scar model, and migrated into the scar tissue. (A) Frozensection analysis and hematoxylin and eosin (HE) staining of scars 4 weeks after model operation. The green fluorescence in the frozen sections indicated the presence of the transplanted mesenchymal stem cells (MSCs) that migrated into the scar tissue. Scale bar, $200 \mu$ m. (B) Representative cultured MSCs that were isolated from the scar tissue. Scale bar, $200 \mu \mathrm{m}$. Ctrl, control; GFP, green fluorescent protein.

MSCs on HTS formation, suggesting that p53 may be essential for MSCs to prevent HTS formation.

\section{TRRI and $\alpha-S M A$ in fibroblast were downregulated by MSCs in a p53-dependent manner}

Transformation of fibroblast to myofibroblast is an important step in HTS development. During the differentiation, fibroblastic cells lose their migratory phenotype and become sessile. Previous studies have shown that the differentiation of scar fibroblast relies on transforming growth factor-beta 1 (TGF- $\beta 1$ ) and $\alpha$-SMA [25]. The T $\beta R I$ could recognize and bind to TGF- $\beta 1$ complex in the fibroblasts, and activate the following signaling pathways. We found that conditioned medium from control shRNA-transduced MSCs significantly downregulated T $\beta R I$ and $\alpha$-SMA in both mRNA (Figure 4A) and protein (Figure 4B,C) levels, while the conditioned medium from p53 shRNAtransduced MSCs did not affect the expression of T $\beta R I$ and $\alpha$-SMA. Our results indicated that the transformation of fibroblast to myofibroblast could be inhibited by MSCs through paracrine in a p53-dependent manner.
Elevated level of nitric oxide produced by p53-deficient MSCs may be responsible for the promotion of HTS fibroblast proliferation

It was reported that p53 could decrease iNOS expression in certain murine fibroblasts and MSCs by downregulating iNOS promoter activity [26]. After treatment with interferon gamma and TNF $\alpha$, iNOS mRNA expression in p53 knockdown MSCs was significantly increased compared with control shRNA-transduced MSCs (Figure 5A). NO production was enhanced in p53 knockdown MSCs compared with control shRNA-transduced MSCs (Figure 5B). To examine the roles of NO in the proliferation of HTS fibroblasts, L-NMMA - an iNOS inhibitor - was used to manipulate the NO level in the conditioned medium from MSCs with or without p53 knockdown. L-NMMA significantly ablated the promotion of fibroblast proliferation caused by the conditioned medium from p53 knockdown MSCs (Figure 5C). These results suggested that the elevated production of iNOS and NO by p53 deficiency MSCs may be responsible for the promotion of HTS fibroblast proliferation. 

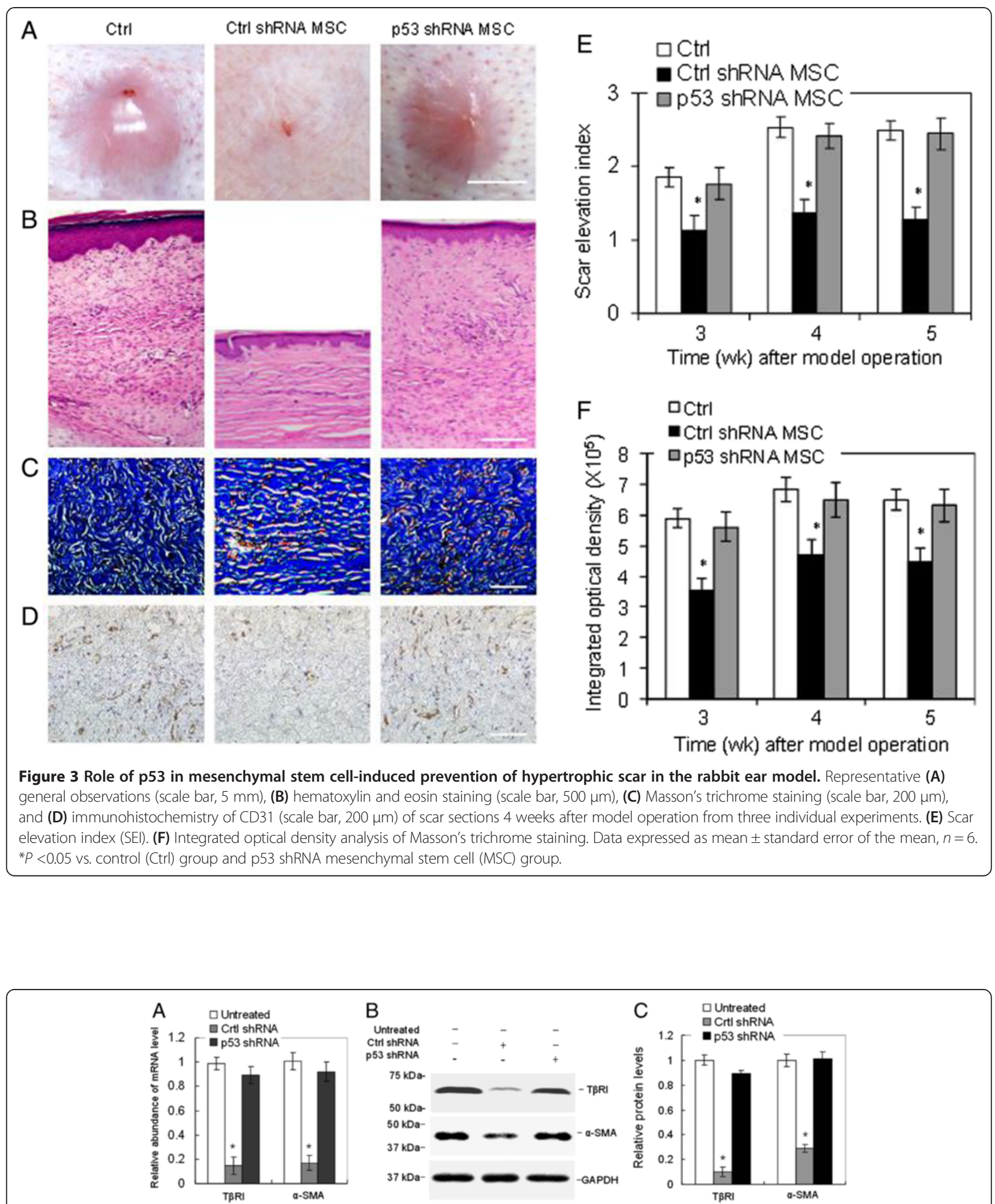

Figure 4 T $\beta R I$ and a-SMA of fibroblasts were downregulated by mesenchymal stem cells in a p53-dependent manner. Fibroblasts

derived from rabbit ear hypertrophic scar were treated with or without conditioned medium from control shRNA mesenchymal stem cells (MSCs) or p53 shRNA MSCs for 48 hours. The $\mathbf{( A )}$ mRNA and $\mathbf{( B , C )}$ protein levels of TBRI and a-SMA were determined by real-time PCR and western blot. $n=3,{ }^{*} P<0.05$ vs. untreated group, p53 shRNA group. Data shown are representative of three individual experiments. a-SMA, alpha smooth muscle actin; GAPDH, glyceraldehyde 3-phosphate dehydrogenase; TRRI, transforming growth factor-beta receptor 1. 

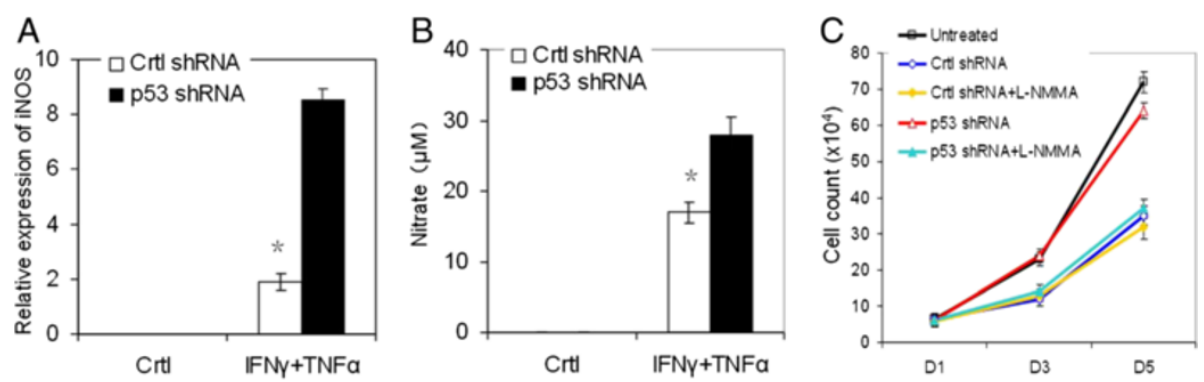

Figure 5 Elevated nitric oxide level produced by mesenchymal stem cells with p53 deficiency may be responsible for the proliferation promotion of hypotrophic scar fibroblasts. Mesenchymal stem cells (MSCs) with or without p53 knockdown were treated with or without interferon gamma (IFN $)$ and tumor necrosis factor alpha (TNFa) $(10 \mathrm{ng} / \mathrm{ml}$ each) for 24 hours. (A) Inducible nitric oxide synthase (iNOS) mRNA was measured by real-time PCR. (B) Total nitrate was measured by Griess. (C) Conditioned medium from MSCs treated with IFNY and TNFa for 24 hours was added to cultured hypertrophic scar fibroblasts with or without treatment with $1 \mathrm{mM} \mathrm{N} N^{G}$-monomethyl-L-arginine acetate salt (L-NMMA). Cells were trypsinized and counted 48 hours later on the indicated days. $n=3,{ }^{*} P<0.05$ vs. p53 shRNA group. Data shown are representative of three individual experiments. Ctrl, control.

\section{Discussion}

The formation of HTS is considered a result of aberrations of wound healing following any injury to the deep dermis, including burn injury, lacerations, abrasions, surgeries, and so on. Any factor that delays wound healing would increase the chance of hypertrophic scarring. On the contrary, if the process of wound healing is accelerated, the occurrence or degree of scarring would be reduced $[2,3]$. As known, the time to heal is the most important factor to predict the development of HTS in a burn wound. If healing occurred between 14 and 21 days, only one-third of anatomical sites became hypertrophic. However, $78 \%$ of the sites developed HTS if the burn wound healed after 21 days. The time to heal could influence many other factors involved in HTS formation, including transformation of the wound clot into granulation tissue, which requires a delicate balance between extracellular matrix protein deposition and degradation [27]. Stem cells have been regarded as a potentially useful agent for promoting wound healing. Specifically, bone marrow MSCs were shown to improve wound healing in several studies $[4,5]$. Human bone marrow MSCs prevented HTS formation in the rabbit ear HTS model via inflammatory regulation and secretion of an anti-inflammatory protein, TNF $\alpha$-stimulated gene/protein 6 [28]. The immune-modulating properties, especially the immune-suppression effect, of MSCs have made it widely applicable, although the mechanisms remain unclear. Our current study shows that transplantation of MSCs via the rabbit ear artery is an effective approach for MSC engraftment and migration in the rabbit HTS model. Furthermore, MSCs engraftment significantly reduced the formation of rabbit ear HTS, suggesting that MSCs may have potential clinical applications in modulating the process of wound healing. In addition, we also found less infiltrated inflammatory cells in the scar tissue with HE staining in the control shRNA-transduced MSC group. Although we focus on p53 in the present study, further investigation about inflammatory modulation of MSCs would be performed in the future.

The aberration of gene expression in HTS has been well studied, but the most critical genes in HTS progression remain undefined [29-31]. In the HTS tissue, p53 protein was reported to have a higher level compared with normal dermis tissue [18], but the roles of p53 in HTS have not been fully investigated, and little is known about p53 gene status in MSCs in the HTS tissue. Investigators recently found that MSCs could interact with other types of cells in the microenvironment, resulting in gene expression or even the phenotype changes of those cells; for example, the epithelial-mesenchymal transition of tumor cells [32-35]. Interestingly, the gene status of p53 in the MSCs has been reported to play an essential role in tumor development [12]. We therefore hypothesized that the p53 in MSCs plays an important role in wound healing and then the development of HTS. To test this hypothesis, we established MSCs with stable p53 knockdown, and transplanted them into the rabbit ear HTS model. We found that the engraftment of p53-deficient MSCs can hardly inhibit the scar formation, suggesting that p53 plays an essential role in preventing HTS formation by MSCs. According to this result, the higher expression level of p53 in the HTS tissue as previously reported may be the outcome of self-regulation of anti-scarring in the microenvironment.

Central to the formation of HTS tissue is the transformation of HTS fibroblast to myofibroblast [36], the latter being responsible for the excessive deposition and irreversible remodeling of the extracellular matrix [37]. The TGF- $\beta 1$ signaling pathway and $\alpha$-SMA are involved in the phenotype transformation $[38,39]$. Our results showed that wild-type MSCs downregulated the expression of T $\beta R I$ and $\alpha$-SMA at both the mRNA and protein levels in a paracrine manner, while the p53-deficient MSCs did not affect the expression of these two genes. These data 
suggest that the inhibition of fibroblast transformation to myofibroblast may account for the anti-scarring outcome by MSC engraftment, in a p53-dependent manner. However, the mechanisms of MSCs regulating the expression of T $\beta R I$ and $\alpha$-SMA of fibroblasts are still unclear. Further investigations on these mechanisms will be helpful for understanding the role of MSCs in HTS formation.

The roles of oxidative/nitrosative stress in cell proliferation are controversial [40-42]. Our previous studies showed that the oxidization level in HTS tissue is elevated compared with normal tissue [43]. In this study, we used interferon gamma and TNF $\alpha$ as stimulation for MSCs to produce NO before collecting conditioned medium. Our data suggest that the elevated level of NO produced by MSCs with p53 knockdown is involved in the proliferation promotion of HTS fibroblasts, and MSCs could inhibit the transformation of HTS fibroblast to myofibroblast in a p53-dependent manner.

\section{Conclusions}

The results in the current study demonstrated that wildtype MSC engraftment could inhibit the formation of HTS, in a p53-dependent manner. This effect might be achieved through downregulating T $\beta R I$ and $\alpha$-SMA gene expression, and inhibiting the transformation of HTS fibroblast to myofibroblast through reducing NO production.

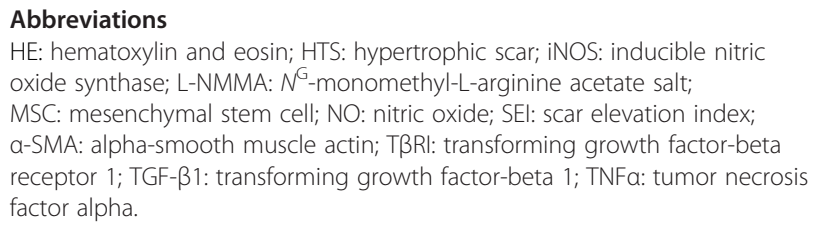

\section{Competing interests}

The authors declare that they have no competing interests.

\section{Authors' contributions}

Y-LL and F-MD conceived and designed the experiments. W-HL, Y-LL, JS, Y-HL, N-NZ, Y-ML, T-JH and H-RL performed the experiments. W-HL, Y-LL, JS, YT and F-MD analyzed the data. H-RL, T-JH, Y-ML and F-MD contributed reagents/materials/analysis tools. $Y-L L$ and $W-H L$ wrote the paper. All authors read and approved the final version of the manuscript.

\section{Acknowledgements}

This work was supported by grants from the Key Discipline Construction Project of the First Affiliated Hospital of Chengdu Medical College (SSZDXK-001) and the Applied Basic Research Project of Sichuan Province Science Technology Department (2011JYZ035). The authors thank Dr Changhong Shi for his help on the animal model construction.

\section{Author details}

${ }^{1}$ Department of Burns and Plastic Surgery, First Affiliated Hospital of Chengdu Medical College, Chengdu, China. ${ }^{2}$ Department of Pathology, Chengdu Medical College, 783 Xindu Avenue, Xindu District, Chengdu 610500, China. ${ }^{3}$ Plastic and Cosmetic Surgery Center, Subei People's Hospital of Jiangsu Province, Yangzhou, China.

Received: 9 June 2014 Revised: 10 December 2014

Accepted: 11 December 2014 Published: 15 December 2014

\section{References}

1. Viera MH, Amini S, Valins W, Berman B: Innovative therapies in the treatment of keloids and hypertrophic scars. J Clin Aesthet Dermatol 2010, 3:20-26.

2. Bloemen MC, van der Veer WM, Ulrich MM, van Zuijlen PP, Niessen FB, Middelkoop E: Prevention and curative management of hypertrophic scar formation. Burns 2009, 35:463-475.

3. Gabriel V: Hypertrophic scar. Phys Med Rehabil Clin N Am 2011, 22:301-310.

4. Ding J, Ma Z, Shankowsky HA, Medina A, Tredget EE: Deep dermal fibroblast profibrotic characteristics are enhanced by bone marrowderived mesenchymal stem cells. Wound Repair Regen 2013, 21:448-455.

5. Wu Y, Wang J, Scott PG, Tredget EE: Bone marrow-derived stem cells in wound healing: a review. Wound Repair Regen 2007, 15:S18-S26.

6. Uccelli A, Moretta L, Pistoia $V$ : Mesenchymal stem cells in health and disease. Nat Rev Immunol 2008, 8:726-736.

7. Bergfeld SA, DeClerck YA: Bone marrow-derived mesenchymal stem cells and the tumor microenvironment. Cancer Metastasis Rev 2010, 29:249-261.

8. Studeny M, Marini FC, Champlin RE, Zompetta C, Fidler IJ, Andreeff M: Bone marrow-derived mesenchymal stem cells as vehicles for interferon-beta delivery into tumors. Cancer Res 2002, 62:3603-3608

9. Chen JS, Wong WW, Gurtner GC: Therapeutic potential of bone marrowderived mesenchymal stem cells for cutaneous wound healing Front Immunol 2012, 3:192.

10. Singer NG, Caplan Al: Mesenchymal stem cells: mechanisms of inflammation. Annu Rev Pathol 2011, 6:457-478.

11. Lam MT, Nauta A, Meyer NP, Wu JC, Longaker MT: Effective delivery of stem cells using an extracellular matrix patch results in increased cell survival and proliferation and reduced scarring in skin wound healing. Tissue Eng Part A 2013, 19:738-747.

12. Huang $Y$, Yu P, Li W, Ren G, Roberts Al, Cao W, Zhang X, Su J, Chen $X$, Chen Q, Shou P, Xu C, Du L, Lin L, Xie N, Zhang L, Wang Y, Shi Y: p53 regulates mesenchymal stem cell-mediated tumor suppression in a tumor microenvironment through immune modulation. Oncogene 2013, 33:3830-3838.

13. Ennis WJ, Sui A, Bartholomew A: Stem cells and healing: impact on inflammation. Adv Wound Care (New Rochelle) 2013, 2:369-378.

14. Li W, Ren G, Huang Y, Su J, Han Y, Li J, Chen X, Cao K, Chen Q, Shou P, Zhang L, Yuan ZR, Roberts Al, Shi S, Le AD, Shi Y: Mesenchymal stem cells: a double-edged sword in regulating immune responses. Cell Death Differ 2012, 19:1505-1513.

15. Ren G, Zhang L, Zhao X, Xu G, Zhang Y, Roberts Al, Zhao RC, Shi Y: Mesenchymal stem cell-mediated immunosuppression occurs via concerted action of chemokines and nitric oxide. Cell Stem Cell 2008, 2:141-150.

16. Penn JW, Grobbelaar AO, Rolfe KJ: The role of the TGF-beta family in wound healing, burns and scarring: a review. Int J Burns Trauma 2012, 2:18-28.

17. De Felice B, Garbi C, Santoriello M, Santillo A, Wilson RR: Differential apoptosis markers in human keloids and hypertrophic scars fibroblasts. Mol Cell Biochem 2009, 327:191-201.

18. Tanaka A, Hatoko M, Tada H, lioka H, Niitsuma K, Miyagawa S: Expression of p53 family in scars. J Dermatol Sci 2004, 34:17-24.

19. Moon JH, Kwak SS, Park G, Jung HY, Yoon BS, Park J, Ryu KS, Choi SC, Maeng I, Kim B, Jun EK, Kim S, Kim A, Oh S, Kim H, Kim KD, You S: Isolation and characterization of multiipotent human keloid-derived mesenchymal-like stem cells. Stem Cells Dev 2008, 17:713-724.

20. Kryger ZB, Sisco M, Roy NK, Lu L, Rosenberg D, Mustoe TA: Temporal expression of the transforming growth factor-Beta pathway in the rabbit ear model of wound healing and scarring. J Am Coll Surg 2007, 205:78-88.

21. Liang Q, Liu S, Han P, Li X, Li X, Zhao Y, Liang Y, Deng Z, Jin Y: Micronized acellular dermal matrix as an efficient expansion substrate and delivery vehicle of adipose-derived stem cells for vocal fold regeneration. Laryngoscope 2012, 122:1815-1825.

22. Kloeters O, Tandara A, Mustoe TA: Hypertrophic scar model in the rabbit ear: a reproducible model for studying scar tissue behavior with new observations on silicone gel sheeting for scar reduction. Wound Repair Regen 2007, 15:S40-S45.

23. Miranda KM, Espey MG, Wink DA: A rapid, simple spectrophotometric method for simultaneous detection of nitrate and nitrite. Nitric Oxide 2001, 5:62-71. 
24. Ruan GP, Han YB, Wang TH, Xing ZG, Zhu XB, Yao X, Ruan GH, Wang JX, Pang RQ, Cai XM, He J, Zhao J, Pan XH: Comparative study among three different methods of bone marrow mesenchymal stem cell transplantation following cerebral infarction in rats. Neurol Res 2013, 35:212-220

25. Wipff PJ, Hinz B: Integrins and the activation of latent transforming growth factor beta1 - an intimate relationship. Eur J Cell Biol 2008, 87:601-615.

26. Forrester K, Ambs S, Lupold SE, Kapust RB, Spillare EA, Weinberg WC, Felley-Bosco E, Wang XW, Geller DA, Tzeng E, Billiar TR, Harris CC: Nitric oxide-induced $\mathrm{p} 53$ accumulation and regulation of inducible nitric oxide synthase expression by wild-type p53. Proc Natl Acad Sci U S A 1996 93:2442-2447.

27. Cubison TC, Pape SA, Parkhouse N: Evidence for the link between healing time and the development of hypertrophic scars (HTS) in paediatric burns due to scald injury. Burns 2006, 32:992-999.

28. Liu S, Jiang L, Li H, Shi H, Luo H, Zhang Y, Yu C, Jin Y: Mesenchymal stem cells prevent hypertrophic scar formation via inflammatory regulation when undergoing apoptosis. J Invest Dermatol 2014, 134:2648-2657.

29. Lewis WH, Sun KK: Hypertrophic scar: a genetic hypothesis. Burns 1990 16:176-178.

30. Thompson CM, Hocking AM, Honari S, Muffley LA, Ga M, Gibran NS: Genetic risk factors for hypertrophic scar development. J Burn Care Res 2013, 34:477-482.

31. Bayat A, Bock O, Mrowietz U, Ollier WE, Ferguson MW: Genetic susceptibility to keloid disease and hypertrophic scarring: transforming growth factor beta1 common polymorphisms and plasma levels. Plast Reconstr Surg 2003, 111:535-543. discussion 544-546.

32. Mandel K, Yang Y, Schambach A, Glage S, Otte A, Hass R: Mesenchymal stem cells directly interact with breast cancer cells and promote tumor cell growth in vitro and in vivo. Stem Cells Dev 2013, 22:3114-3127.

33. Ryu HS, do Park J, Kim HH, Kim WH, Lee HS: Combination of epithelialmesenchymal transition and cancer stem cell-like phenotypes has independent prognostic value in gastric cancer. Hum Pathol 2012, 43:520-528.

34. Wang $H$, Zhang G, Zhang $H$, Zhang F, Zhou B, Ning F, Wang HS, Cai SH, Du J: Acquisition of epithelial-mesenchymal transition phenotype and cancer stem cell-like properties in cisplatin-resistant lung cancer cells through AKT/beta-catenin/Snail signaling pathway. Eur J Pharmacol 2014, 723:156-166.

35. Strassburg S, Hodson NW, Hill PI, Richardson SM, Hoyland JA: Bi-directional exchange of membrane components occurs during co-culture of mesenchymal stem cells and nucleus pulposus cells. PLoS One 2012, 7:e33739.

36. Ennis WJ, Meneses P: Wound healing at the local level: the stunned wound. Ostomy Wound Manage 2000, 46:39S-48S. quiz 49S-50S.

37. Wynn TA: Cellular and molecular mechanisms of fibrosis. J Pathol 2008 214:199-210.

38. Guo W, Shan B, Klingsberg RC, Qin X, Lasky JA: Abrogation of TGF-beta1induced fibroblast-myofibroblast differentiation by histone deacetylase inhibition. Am J Physiol Lung Cell Mol Physiol 2009, 297:L864-L870.

39. Garrett Q, Khaw PT, Blalock TD, Schultz GS, Grotendorst GR, Daniels JT: Involvement of CTGF in TGF-beta1-stimulation of myofibroblast differentiation and collagen matrix contraction in the presence of mechanical stress. Invest Ophthalmol Vis Sci 2004, 45:1109-1116.

40. Kurz T, Gustafsson B, Brunk UT: Intralysosomal iron chelation protects against oxidative stress-induced cellular damage. FEBS J 2006, 273:3106-3117.

41. Radosavljevic T, Mladenovic D, Vucevic D, Vukicevic RJ: The role of oxidative/nitrosative stress in pathogenesis of paracetamol-induced toxic hepatitis. Med Pregl 2010, 63:827-832
42. Svegliati Baroni G, D’Ambrosio L, Ferretti G, Casini A, Di Sario A, Salzano R, Ridolfi F, Saccomanno S, Jezequel AM, Benedetti A: Fibrogenic effect of oxidative stress on rat hepatic stellate cells. Hepatology 1998, 27:720-726.

43. Liu YL, Liu YM, Sun J, Wen K: Significance of glutathione and coenzyme I in pathological scars. Zhongguo Mei Rong Zheng Xing Wai Ke Za Zhi 2009, 20:501-503.

doi:10.1186/scrt526

Cite this article as: Liu et al:: Mesenchymal stem cell-mediated suppression of hypertrophic scarring is $\mathrm{p} 53$ dependent in a rabbit ear model. Stem Cell Research \& Therapy 2014 5:136.

\section{Submit your next manuscript to BioMed Central and take full advantage of:}

- Convenient online submission

- Thorough peer review

- No space constraints or color figure charges

- Immediate publication on acceptance

- Inclusion in PubMed, CAS, Scopus and Google Scholar

- Research which is freely available for redistribution

Submit your manuscript at www.biomedcentral.com/submit
C) Biomed Central 